\title{
Bio-molecular event extraction by integrating multiple event- extraction systems
}

\author{
AMIT MAJUMDER $^{1, *}$, ASIF EKBAL $^{2}$ and SUDIP KUMAR NASKAR ${ }^{3}$ \\ ${ }^{1}$ Techno India University, Kolkata 700091, India \\ ${ }^{2}$ Indian Institute of Technology, Patna, India \\ ${ }^{3}$ Jadavpur University, Kolkata, India \\ e-mail: jobamit48@yahoo.co.in; asif@iitp.ac.in; sudip.naskar@gmail.com
}

MS received 19 March 2018; accepted 7 July 2018; published online 2 January 2019

\begin{abstract}
Event extraction from biomedical text is a very important task in text mining and natural language processing. The overall task involves finding event-related expressions, classifying these into predefined categories and attaching arguments to these events. We perform event detection and event classification in one step using an ensemble of classifiers. For event argument extraction, we also use an ensemble of classification models. Our base models are developed using supervised machine learning that makes use of statistical, contextual and syntactic features. Our experimental result on the benchmark datasets of BioNLP-2011 shared task shows the recall, precision and $F$-measure values of $51.20 \%, 65.78 \%$ and $57.58 \%$, respectively.
\end{abstract}

Keywords. Event extraction; machine learning; dependency graph; genetic algorithm; class-weight tuning.

\section{Introduction and background}

Biomedical documents in electronic form are growing rapidly in the Internet. Hence, the cost of retrieving relevant information is increasing and inferring scientific knowledge from this huge information is becoming a challenging task. Success of text mining (TM) is evident from the organization of different shared-task evaluation campaigns, such as those organized in the MUC [1], TREC [2], $\mathrm{ACE}^{1}$, etc. Some of the very popular bio-TM evaluation challenges include the TREC Genomics track [2], JNLPBA ${ }^{2}$, LLL [3] and BioCreative [4]. All these tasks were dedicated towards coarse-grained information extraction. There is a recent trend for fine-grained information extraction from the text [5], which is addressed in consecutive TM challenges [6-9].

Literature shows various techniques for event extraction. They cover supervised approach [10], rule-based approach [11], MSTParser-based approach [12], co-reference-based approach [13] and very recently deep-learning-based techniques [14]. Our event-extraction system is based on an ensemble model that combines several event-extraction systems. Individual event-extraction systems are based on supervised multi-class classification approach. Let us consider the following example sentence for better explanation: "BMP-6 inhibits growth of mature human B cells; induction of Smad phosphorylation and upregulation of IdI". In this example sentence, the tokens BMP-6 and $I d I$ are

*For correspondence named entities, i.e., proteins. The token upregulation is an event trigger of type Positive_regulation and argument of this trigger is $I d l$. The event expression generated from this example sentence is shown in table 1.

In this paper we propose an effective technique of event extraction by combining several individual models. Eventextraction systems are not so effective individually in extracting bio-molecular events. Individual systems have their own strengths and weaknesses and they tend to perform well on some cases while performing poorly on other cases. Therefore, we integrate multiple event-extraction systems in a bid to improve the overall performance.

The overall task involves detecting event expressions, classifying them into pre-defined categories and attaching arguments to the events. The events are classified into nine potential events. Among them, five are simple, which correspond to gene expression, transcription, protein catabolism, phosphorylation and localization. The remaining four events, namely binding, regulation, positive_regulation and negative_regulation, are relatively complex. For simple events we have only a single primary theme, which is usually a protein. However, a complex event can include a theme as well as a cause argument. These theme and cause arguments can be either proteins or events. Moreover, number of theme arguments could also vary. We evaluate

\footnotetext{
$\overline{{ }^{1} \text { http://www.itl.nist.gov/iad/mig/tests/ace/. }}$ ${ }^{2} \mathrm{http} / / / \mathrm{www}$.geniaproject.org/shared-tasks/bionlp-jnlpba-shared-task2004.
} 
Table 1. Example event annotation. $\mathrm{T} 1$ and $\mathrm{T} 2$ are the protein annotations for the sentence "BMP-6 inhibits growth of mature human B cells; induction of Smad phosphorylation and upregulation of Idl."

\begin{tabular}{lc}
\hline T1 & (Protein, BMP-6) \\
T2 & (Protein, Id1) \\
T3 & (Positive_regulation, upregulation) \\
E1 & (Type/class: T3, theme: T2) \\
\hline
\end{tabular}

our proposed system on the benchmark dataset of BioNLP 2011 shared task.

\section{Proposed approach}

Our proposed system follows an ensemble architecture where we combine various individual models. For this purpose, at first we develop four different event-extraction systems and then combine the outputs of these individual systems. We depict the overall system architecture in figure 1. Later, we describe the individual event-extraction systems. All these systems made use of the same set of features.

1. System-1 and System-2: These two systems are based on multi-class support vector machine (SVM) with classweight tuning [15]. Both of these systems use the same set of features; however, they use different class weights. These class weights are tuned using a genetic algorithm (GA) [16].

2. System-3: This system is based on LinearSVC ${ }^{3}$, an implementation of SVM using liblinear. Liblinear is a linear classifier for data with millions of instances and features.

3. System-4: This system is based on a Stacked Generalization (Stacking) approach [17], which makes use of three classification algorithms, namely SVM, stochastic gradient descent (SGD) and logistic regression (LR). They are used to implement the base classifiers. Linear SVM is used as a meta-classifier.

4. Ensemble of various models: There are different categories of event expressions like gene_expression, binding, regulation, etc. For evaluation we use BioNLP-2011 evaluation scheme, which generates recall, precision and $F$-score for each type of event expression. While one system shows good performance for one class, others perform well on the other classes. Therefore, we combine the outputs of different systems on the basis of $F$-score value per class. This combination process is an iterative method, as shown in figure 2. In every iteration we combine a pair of systems by taking the best

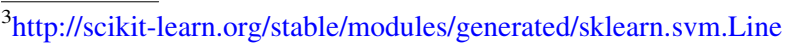
arSVC.html.
}

results from the two systems and thus obtain a new system's output.

\subsection{An example showing the combination process}

To explain the algorithm about how the outputs of different systems are actually combined, let us consider the following example sentence "BMP-6 inhibits growth of mature human B cells; induction of Smad phosphorylation and upregulation of IdI". We consider two systems SO and S1, whose outputs are shown in tables 2 and 3 , respectively. Figure 3 shows the step-by-step operation for combining the outputs. Result of combining the outputs of systems $S O$ and $S 1$ have been shown in table 4 .

Among the four systems, two systems (System-1 and System-2) are based on multi-class SVM followed by classweight tuning by a GA-based approach. The method of class-weight tuning is described later.

\subsection{A brief overview of $G A$}

GAs are randomized search and optimization techniques. They are based on the principles of evolution and natural genetics. GAs provide near-optimal solutions for an objective function of an optimization problem. In GAs, the parameters of the search space are encoded in the form of strings called chromosomes. A collection of such chromosomes is called a population. Initially, a random population is created, which represents different points in the search space. An objective or a fitness function is associated with each chromosome that represents the goodness of the chromosome. Using survival of the fittest principle, a few of the chromosomes are selected and each is assigned a number of copies that go into the mating pool. Crossover and mutation operators are applied on these chromosomes to yield a new generation of chromosomes. The process of selection, crossover and mutation continues either for a predefined number of generations or until a termination condition is satisfied.

\subsection{Class-weight tuning for performance improvement}

When we apply SVM algorithm to classify data, it generates confidence score for each class label. Confidence score of each class is multiplied by a corresponding weight factor, which is produced by GA. The class receiving the highest score is considered as the final predicted class label. The class label for an instance $\mathrm{E}$ is predicted based on the formula in Eq. (1), where $C$ refers to the set of classes:

$$
\hat{c}_{E}=\operatorname{argmax}_{c_{i} \in C}\left\{\text { ConfidenceScore }_{c_{i}}^{E} * \text { Weight }_{c_{i}}^{E}\right\} .
$$




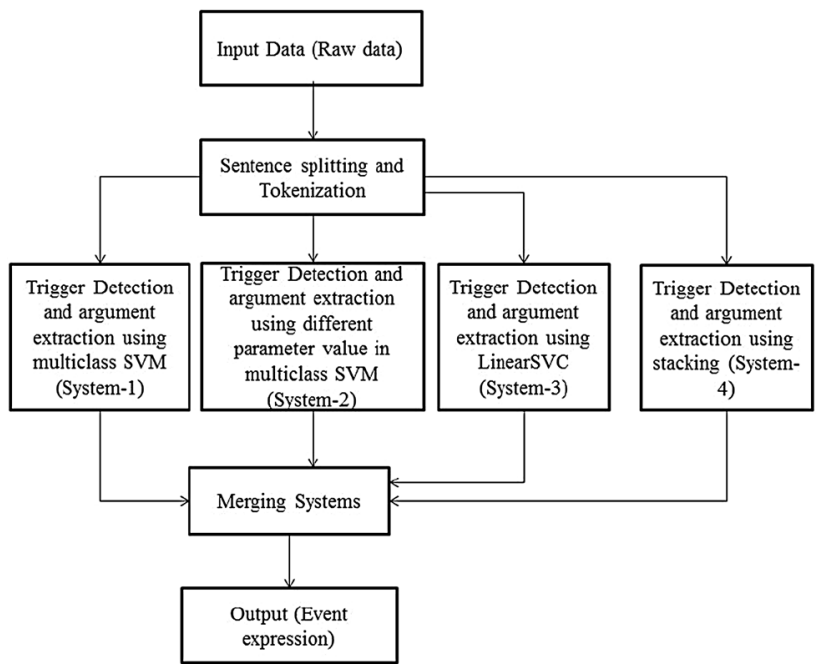

Figure 1. Overall system architecture.

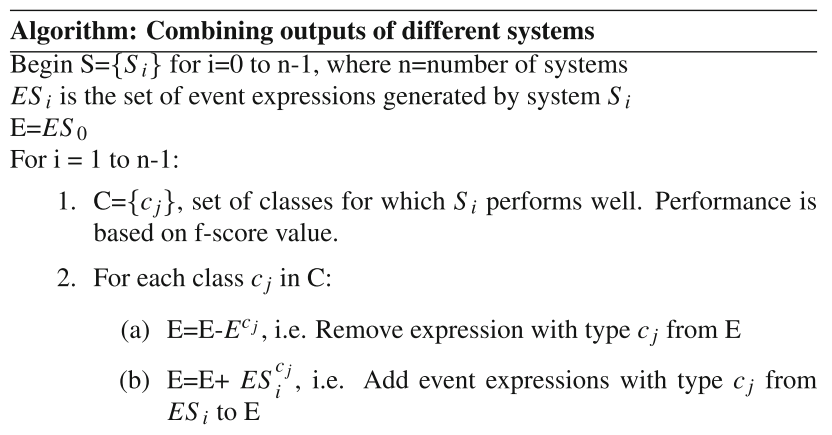

End

Figure 2. Combining outputs of different systems.

Table 2. System SO.

\begin{tabular}{lc}
\hline T1 & (Protein, BMP-6) \\
T2 & (Protein, Id1) \\
T3 & (Positive_regulation, upregulation) \\
E1 & (Type/class: T3, theme: T1) \\
\hline
\end{tabular}

Table 3. System $S 1$.

\begin{tabular}{lc}
\hline T1 & (Protein, BMP-6) \\
T2 & (Protein, Id1) \\
T3 & (Positive_regulation, upregulation) \\
T4 & (Phosphorylation, phosphorylation) \\
E2 & (Type/class: T3, theme: T2) \\
E3 & (Type/class: T4, theme: T2) \\
\hline
\end{tabular}

Here, ConfidenceScore $c_{c_{i}}^{E}$ represents the confidence score of class label $c_{i}$ for the instance $\mathrm{E}$ and Weight ${ }_{c_{i}}^{E}$ represents the weight value chosen for class label $c_{i}$. Figure 4 shows an example for encoding the problem of chromosome representation to be used by GA. In the figure, $C_{0}-C_{N-1}$ are $n$ class-labels. The value in each cell represents the weight of the corresponding class label mentioned above it.

Fitness computation: Each chromosome is represented by the weights of the class labels as shown in figure 4 . We create classifiers by applying weights on confidence scores for the class labels using the formula in Eq. (1). The classifier is evaluated on the development dataset to compute recall, precision and $F$-measure values. We consider $F$ measure as the fitness of the respective chromosome.

\section{Features}

We use different types of features that include wordforms, named entity (NE), stem, chunk, part-of-speech (PoS), bag-of-words (BOW), bi-grams, tri-grams and linear features. We also use several forms of syntactic features generated from the dependency parser [18]. These features include dependency path, dependency labels of incoming edges and outgoing edges, and dependency chain features [17]. Dependency features are very important for event extraction. Figure 5 shows the dependency graph for the sentence "BMP-6 inhibits growth of mature human B cells; induction of Smad phosphorylation and upregulation of IdI", generated by the CharniakMcCloskey parser [18]. In the graph, an edge label represents the dependency relation between two nodes. Each node in the graph is labelled by a number, which represents a word appearing in that position (0-based index) of the sentence. For example, the node labelled with number 0 indicates the word BMP-6 and node labelled with number 1 indicates the word inhibits.

In the graph, nodes 0 and 16 represent proteins (i.e., NE) as specified in the training dataset. In the feature value, NE is denoted by _NAMED_ENT.

Edges in a dependency graph are directed arcs. Each edge connects two nodes. Nodes represent words along with other information like PoS tags of the words. A node can be connected to two types of edges: one is in-type (or incoming) edges, which are incident on the node, and the other type is out-type (or outgoing) edges, which emanate from the node. Following features are generated from the dependency graph.

Features for in-type edges:

These features are generated using information of incoming edges for a node in the graph. These features include edge type, PoS of source node, text of source node, etc. for the incoming edges.

Features for out-type edges:

These features are generated using information of outgoing edges for a node in the graph. These features include edge type, PoS of destination node, text of destination node, etc. for the outgoing edges. 


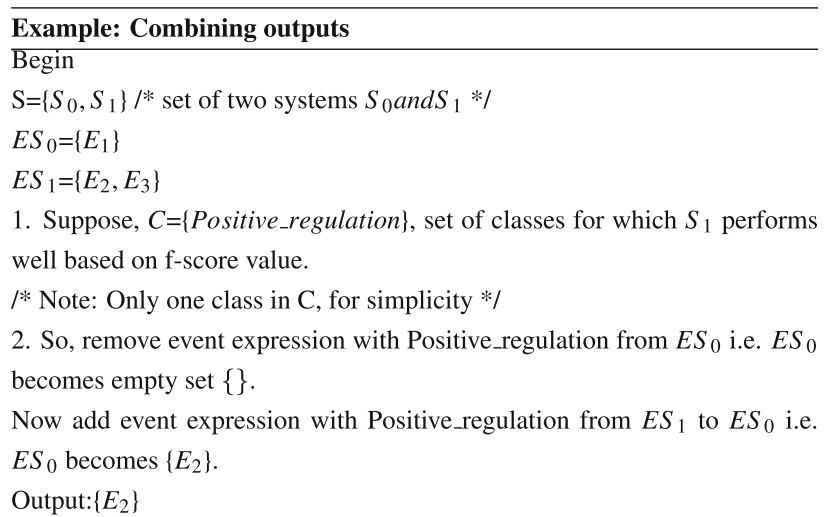

Figure 3. Combining outputs of different systems.

Table 4. Output after combining systems $S O$ and $S 1$.

\begin{tabular}{lc}
\hline T1 & (Protein, BMP-6) \\
T2 & (Protein, Id1) \\
T3 & (Positive_regulation, upregulation) \\
E1 & (Type/class: T3, theme: T2) \\
\hline
\end{tabular}

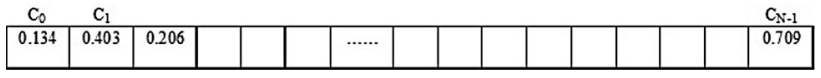

Figure 4. Chromosome representation.

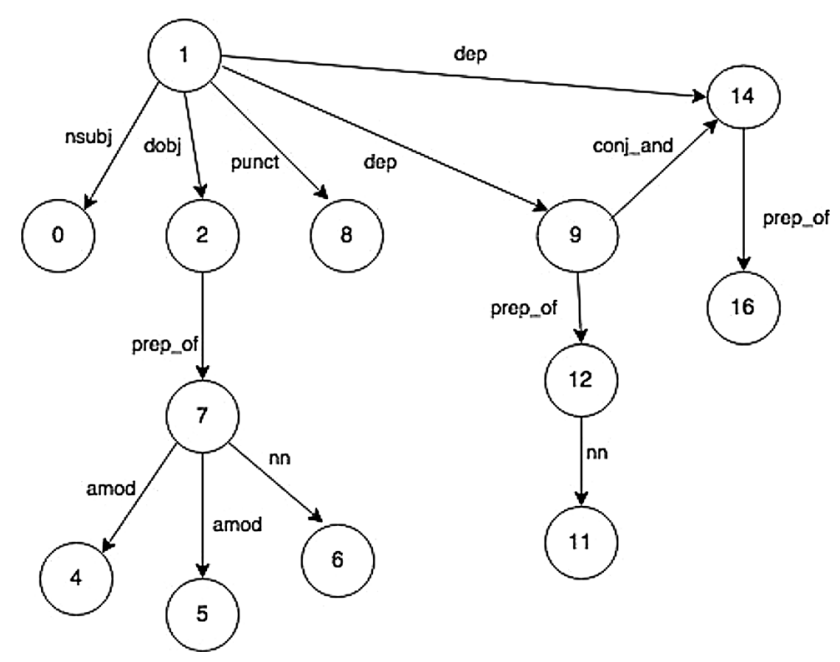

Figure 5. Dependency graph for the example sentence "BMP-6 inhibits growth of mature human B cells; induction of Smad phosphorylation and upregulation of Id1."

Dependency chain: These features are syntactic dependencies up to a certain depth limit, starting from a token of interest. In our experiment we use the depth of limit three.

\section{Experimental results and analysis}

We use the BioNLP-ST-2011 datasets and evaluation frameworks ${ }^{4}$ for the experiments. We tuned the system on the development dataset, and used the configurations obtained for final evaluation. The tuned system is evaluated on the test dataset. Statistics of BioNLP-11 dataset for Genia event extraction is shown in table 5.

We perform both trigger detection and classification at the same time. We determine the class weights using a GA-based technique [15] for System-1 and System-2. For GA the following parameter values are set: population size $=30$, number of generations $=40$, selection strategy $=-$ tournament selection, probability of crossover $=0.9$ and probability of mutation $=0.125$. Tables 6 and 7 show the results produced by SVM-based classifier used for building System-1 and System-2. Tables 8 and 9 show the results of LinearSVC and the Stacking approach, respectively. After combining the outputs, we finally obtain the results as shown in table 10. All these evaluation results are reported in the BioNLP-2011 test dataset for Genia event extraction.

From the experimental results it is evident that System-2 produces better performance for Phosphorylation ( $F$-score value $84.62 \%)$, Localization (68.09\%) and Positive_regulation (46.31\%) event types. System-1 is the best for Negative_regulation (43.94\%) type event. On the other hand, the third system (i.e., System-3) is the best for handling the Protein_catabolism.

\subsection{Error analysis}

We have very closely analysed the outputs to find out the errors and the possible reasons of the errors. For event trigger detection, we observe that the system's output is good for Gene expression and Phosphorylation types. However, the classifier is not so good for Binding, Regulation, Positiv_regulation and Negative_regulation type events. One of the reasons may be the less number of training instances for regulatory events. As our architecture follows a pipelined model where the output of one stage is fed to next stage, the error generated in one stage propagates to the next stage. This, in turn, causes errors for argument extraction. For example, the token augmented in one example sentence has been detected as event trigger (type: Positive_regulation) and CD4 has been identified as a cause type argument, though the token augmented is actually not an event trigger. The classifier finds it difficult to disambiguate the cases when a particular instance belongs to more than one type. For example, token transfection originally belongs to two event types, which are Gene expression and

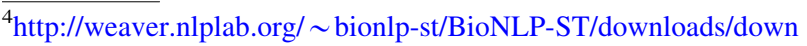
loads.shtml.
} 
Table 5. Statistics of BioNLP-ST 2011 Genia event dataset. Values inside parentheses indicate the number of full articles.

\begin{tabular}{lccc}
\hline Attributes & Training & Development & Test \\
\hline Abstracts+full articles & $908(5)$ & $259(5)$ & $347(5)$ \\
Sentences & 8,759 & 2,954 & 3,437 \\
Proteins & 11,625 & 4,690 & 5,301 \\
Total events & 10,287 & 3,243 & 4,457 \\
\hline
\end{tabular}

Positive_regulation, but our system is unsuccessful in detecting the word as a trigger word. On the other hand, the word Overexpression is originally not a trigger word, but using our system we find that Overexpression is identified as a trigger word of both types Positive regulation and Gene expression.

\subsection{Comparison with the existing systems}

The state-of-the-art system for bio-molecular event extraction is TEES [19]. This system is ranked first place in BioNLP-ST-2009. We compare our experimental results to those of event-extraction system TEES. The official scores of TEES is recall $49.56 \%$, precision $57.65 \%$ and $F$-measure $53.30 \%$ on test dataset. Our system achieves the recall, precision and $F$-measure values of $51.20 \%, 65.78 \%$ and $57.58 \%$, respectively, on the test set. Thus, our system performs better than TEES (4.28\% F-measure gain). The performance that we achieve is better than that of the system FAUST [20] (recall: $49.41 \%$, precision: $64.75 \%$ and $F$-measure: $56.04 \%$ ), which is the best performing system in BioNLP-2011 [7]. Our system outperforms the recently developed system named EventMine [21], which achieved the recall, precision and $F$-measure of $51.25 \%, 64.92 \%$ and $57.28 \%$, respectively.

Table 6. System-1 [approximate span/approximate recursive].

\begin{tabular}{|c|c|c|c|c|c|}
\hline Event class & Gold (match) & Answer (match) & Recall & Prec. & $F$-score \\
\hline Gene_expression & $1002(705)$ & $845(705)$ & 70.36 & 83.43 & 76.34 \\
\hline Transcription & $174(83)$ & $103(83)$ & 47.70 & 80.58 & 59.93 \\
\hline Protein_catabolism & $15(6)$ & $7(6)$ & 40.00 & 85.71 & 54.55 \\
\hline Phosphorylation & $185(156)$ & $186(156)$ & 84.32 & 83.87 & 84.10 \\
\hline Localization & $191(93)$ & $108(93)$ & 48.69 & 86.11 & 62.21 \\
\hline$=[$ SVT - TOTAL $]=$ & $1567(1043)$ & $1249(1043)$ & 66.56 & 83.51 & 74.08 \\
\hline Binding & $491(212)$ & $396(212)$ & 43.18 & 53.54 & 47.80 \\
\hline$==[$ EVT-TOTAL $]==$ & $2058(1255)$ & $1645(1255)$ & 60.98 & 76.29 & 67.78 \\
\hline Regulation & $385(113)$ & 203 (113) & 29.35 & 55.67 & 38.44 \\
\hline Positive_regulation & $1443(528)$ & $894(528)$ & 36.59 & 59.06 & 45.19 \\
\hline Negative_regulation & $571(214)$ & $403(214)$ & 37.48 & 53.10 & 43.94 \\
\hline$==[$ REG-TOTAL $]==$ & $2399(855)$ & $1500(855)$ & 35.64 & 57.00 & 43.86 \\
\hline$==[$ ALL-TOTAL $]==$ & 4457 (2110) & $3145(2110)$ & 47.34 & 67.09 & 55.51 \\
\hline
\end{tabular}

Table 7. System-2 [approximate span/approximate recursive].

\begin{tabular}{|c|c|c|c|c|c|}
\hline Event class & Gold (match) & Answer (match) & Recall & Prec. & $F$-score \\
\hline Gene_expression & $1002(741)$ & $942(741)$ & 73.95 & 78.66 & 76.23 \\
\hline Transcription & $174(79)$ & $106(79)$ & 45.40 & 74.53 & 56.43 \\
\hline Protein_catabolism & $15(6)$ & $7(6)$ & 40.00 & 85.71 & 54.55 \\
\hline Phosphorylation & $185(154)$ & 179 (154) & 83.24 & 86.03 & 84.62 \\
\hline Localization & $191(112)$ & $138(112)$ & 58.64 & 81.16 & 68.09 \\
\hline$=[$ SVT-TOTAL $]=$ & $1567(1092)$ & $1372(1092)$ & 69.69 & 79.59 & 74.31 \\
\hline Binding & $491(186)$ & $305(186)$ & 37.88 & 60.98 & 46.73 \\
\hline$==[$ EVT - TOTAL $]==$ & $2058(1278)$ & 1677 (1278) & 62.10 & 76.21 & 68.43 \\
\hline Regulation & $385(114)$ & 235 (114) & 29.61 & 48.51 & 36.77 \\
\hline Positive_regulation & $1443(552)$ & $941(552)$ & 38.25 & 58.66 & 46.31 \\
\hline Negative_regulation & $571(203)$ & 407 (203) & 35.55 & 49.88 & 41.51 \\
\hline$==[$ REG-TOTAL $]==$ & $2399(869)$ & $1583(869)$ & 36.22 & 54.90 & 43.65 \\
\hline$==[$ ALL-TOTAL $]==$ & 4457 (2147) & $3260(2147)$ & 48.17 & 65.86 & 55.64 \\
\hline
\end{tabular}


Table 8. System-3 [approximate span/approximate recursive].

\begin{tabular}{|c|c|c|c|c|c|}
\hline Event class & Gold (match) & Answer (match) & Recall & Prec. & $F$-score \\
\hline Gene_expression & $1002(748)$ & $943(748)$ & 74.65 & 79.32 & 76.92 \\
\hline Transcription & $174(93)$ & $126(93)$ & 53.45 & 73.81 & 62.00 \\
\hline Protein_catabolism & $15(9)$ & $10(9)$ & 60.00 & 90.00 & 72.00 \\
\hline Phosphorylation & 185 (159) & 208 (159) & 85.95 & 76.44 & 80.92 \\
\hline Localization & $191(103)$ & $131(103)$ & 53.93 & 78.63 & 63.98 \\
\hline$=[$ SVT-TOTAL $]=$ & $1567(1112)$ & 1418 (1112) & 70.96 & 78.42 & 74.51 \\
\hline Binding & $491(170)$ & $356(170)$ & 34.62 & 47.75 & 40.14 \\
\hline$==[$ EVT $-\mathrm{TOTAL}]==$ & 2058 (1282) & $1774(1282)$ & 62.29 & 72.27 & 66.91 \\
\hline Regulation & 385 (117) & 283 (117) & 30.39 & 41.34 & 35.03 \\
\hline Positive_regulation & $1443(592)$ & $1256(592)$ & 41.03 & 47.13 & 43.87 \\
\hline Negative_regulation & $571(229)$ & 475 (229) & 40.11 & 48.21 & 43.79 \\
\hline$==[$ REG-TOTAL $]==$ & 2399 (938) & $2014(938)$ & 39.10 & 46.57 & 42.51 \\
\hline$==[$ ALL-TOTAL $]==$ & $4457(2220)$ & $3788(2220)$ & 49.81 & 58.61 & 53.85 \\
\hline
\end{tabular}

Table 9. System-4 [approximate span/approximate recursive].

\begin{tabular}{|c|c|c|c|c|c|}
\hline Event class & Gold (match) & Answer (match) & Recall & Prec. & $F$-score \\
\hline Gene_expression & $1002(729)$ & $864(729)$ & 72.75 & 84.38 & 78.14 \\
\hline Transcription & $174(88)$ & $124(88)$ & 50.57 & 70.97 & 59.06 \\
\hline Protein_catabolism & $15(7)$ & $8(7)$ & 46.67 & 87.50 & 60.87 \\
\hline Phosphorylation & $185(155)$ & $182(155)$ & 83.78 & 85.16 & 84.47 \\
\hline Localization & $191(98)$ & $114(98)$ & 51.31 & 85.96 & 64.26 \\
\hline$=[$ SVT-TOTAL $]=$ & 1567 (1077) & 1292 (1077) & 68.73 & 83.36 & 75.34 \\
\hline Binding & $491(226)$ & $383(226)$ & 46.03 & 59.01 & 51.72 \\
\hline$==[$ EVT - TOTAL $]==$ & $2058(1303)$ & $1675(1303)$ & 63.31 & 77.79 & 69.81 \\
\hline Regulation & $385(127)$ & $243(127)$ & 32.99 & 52.26 & 40.45 \\
\hline Positive_regulation & $1443(516)$ & 897 (516) & 35.76 & 57.53 & 44.10 \\
\hline Negative_regulation & $571(208)$ & $390(208)$ & 36.43 & 53.33 & 43.29 \\
\hline$==[$ REG-TOTAL $]==$ & $2399(851)$ & $1530(851)$ & 35.47 & 55.62 & 43.32 \\
\hline$==[$ ALL-TOTAL $]==$ & 4457 (2154) & 3205 (2154) & 48.33 & 67.21 & 56.23 \\
\hline
\end{tabular}

Table 10. Combined system [approximate span/approximate recursive].

\begin{tabular}{|c|c|c|c|c|c|}
\hline Event class & Gold (match) & Answer (match) & Recall & Prec. & $F$-score \\
\hline Gene_expression & $1002(752)$ & $912(752)$ & 75.05 & 82.46 & 78.58 \\
\hline Transcription & $174(98)$ & $138(98)$ & 56.32 & 71.01 & 62.82 \\
\hline Protein_catabolism & $15(9)$ & $10(9)$ & 60.00 & 90.00 & 72.00 \\
\hline Phosphorylation & $185(155)$ & $181(155)$ & 83.78 & 85.64 & 84.70 \\
\hline Localization & $191(112)$ & $133(112)$ & 58.64 & 84.21 & 69.14 \\
\hline$=[$ SVT - TOTAL $]=$ & $1567(1126)$ & $1374(1126)$ & 71.86 & 81.95 & 76.57 \\
\hline Binding & $491(241)$ & $423(241)$ & 49.08 & 56.97 & 52.74 \\
\hline$==[$ EVT - TOTAL $]==$ & $2058(1367)$ & 1797 (1367) & 66.42 & 76.07 & 70.92 \\
\hline Regulation & 385 (127) & $243(127)$ & 32.99 & 52.26 & 40.45 \\
\hline Positive_regulation & $1443(554)$ & $949(554)$ & 38.39 & 58.38 & 46.32 \\
\hline Negative_regulation & $571(234)$ & $480(234)$ & 40.98 & 48.75 & 44.53 \\
\hline$==[$ REG-TOTAL $]==$ & $2399(915)$ & $1672(915)$ & 38.14 & 54.72 & 44.95 \\
\hline$==[$ ALL-TOTAL $]==$ & 4457 (2282) & 3469 (2282) & 51.20 & 65.78 & 57.58 \\
\hline
\end{tabular}




\section{Conclusion and future works}

In this paper we propose an efficient technique for event extraction in biomedical text. Initially, various individual models have been developed using various features and machine learning models. The models are then combined together to achieve better accuracy. We have performed our experiments on the Bio-NLP 2009 shared-task datasets. Experiments show that integrating many systems improves overall performance significantly. Comparisons with the other existing techniques show that our model attains the state-of-the-art performance. In our future work, we would like to investigate deep-learning-based models for biomedical event extraction.

\section{References}

[1] Chinchor N 1998 Overview of muc-7. In: Proceedings of the Seventh Message Understanding Conference (MUC-7) Held in Fairfax, Virginia, April 29-May 1

[2] Voorhees E M and Buckland L P editors 2007 Proceedings of the Sixteenth Text REtrieval Conference, TREC 2007, Gaithersburg, Maryland, USA, November 5-9, 2007, volume Special Publication 500-274. National Institute of Standards and Technology (NIST)

[3] Nedellec C 2005 Learning language in logic-genic interaction extraction challenge. In: Proceedings of the 4th Learning Language in Logic Workshop (LLLO5), pp. 31-37

[4] Hirschman L, Krallinger M and Valencia A In: Proceedings of the Second BioCreative Challenge Evaluation Workshop, Centro Nacional de Investigaciones Oncologicas (CNIO)

[5] Kim J D, Ohta T, Pyysalo S, Kano Y and Tsujii J 2009 Overview of bionlp09 shared task on event extraction. In: BioNLP 09: Proceedings of the Workshop on BioNLP, pp. 1-9

[6] Lee H G, Cho H C, Kim M J, Lee J Y, Hong G and Rim H C A multi-phase approach to biomedical event extraction. In: Proceedings of the Workshop on BioNLP, pp. 107-110

[7] Kim J D, Pyysalo S, Ohta T, Bossy R, Nguyen N and Tsujii J 2011 Overview of BioNLP Shared Task 2011, pp. 1-6

[8] Li L, Wang Y and Huang D 2013 Improving feature-based biomedical event extraction system by integrating argument information, pp. 109-115
[9] Kim J D, Wang Y, Colic N, Beak S H, Kim Y H and Song M 2016 Refactoring the genia event extraction shared task toward a general framework for IE-driven KB development. In: Proceedings of the Fourth BioNLP Shared Task Workshop, pp. 23-31

[10] Ozgur A and Radev D R Supervised classification for extracting biomedical events. In: Proceedings of the Workshop on BioNLP, pp. 111-114

[11] Bui Q C, Campos D, van Mulligen E and Kors J A 2013 A fast rule-based approach for biomedical event extraction, pp. 104-108

[12] McClosky D, Surdeanu M and Manning C D 2011 Event extraction as dependency parsing for BioNLP 2011. In: Proceedings of the BioNLP Shared Task 2011 Workshop, pp. 41-45

[13] Yoshikawa K, Riedel S, Hirao T, Asahara M and Matsumoto Y 2010 Coreference based event-argument relation extraction on biomedical text. In: Proceedings of the Semantic Mining in Biomedicine Symposium

[14] Wang J, Li H, An Y, Lin H and Yang Z 2016 Biomedical event trigger detection based on convolutional neural network. Int. J. Data Min. Bioinformat. 15(3): 195-213

[15] Majumder A, Ekbal A and Naskar S K 2017 Feature selection and class-weight tuning using genetic algorithm for bio-molecular event extraction. In: Proceedings of the $N L D B$

[16] Deb K, Pratap A, Agarwal S and Meyarivan T 2002 A fast and elitist multiobjective genetic algorithm: NSGA-ii. IEEE Trans. Evol. Comput. 6(2): 182-197

[17] Majumder A, Ekbal A and Naskar S K 2016 Biomolecular event extraction using a stacked generalization based classifier. In: Proceedings of the 13th International Conference on Natural Language Processing, Varanasi, India, December 2016. NLP Association of India, pp. 55-64

[18] Mcclosky D 2010 Any domain parsing: automatic domain adaptation for natural language parsing. PhD thesis, Providence, RI, USA. AAI3430199

[19] Björne J 2014 Biomedical event extraction with machine learning. $\mathrm{PhD}$ thesis, University of Turku

[20] Riedela S, McCloskyb D, Surdeanub M, McCalluma A and Manning C D 2011 Model combination for event extraction in BioNLP 2011, pp. 51-55

[21] Miwa M, Pyysalo S, Ohta T and Ananiadou S 2013 Wide coverage biomedical event extraction using multiple partially overlapping corpora. BMC Bioinformat. 14(1): 175 